\title{
Atomic-Resolution Investigation of Irradiation-Induced Defects in Silicon Carbide
}

\author{
Chad M. Parish, ${ }^{1}$ Takaaki Koyanagi, ${ }^{1}$ Sosuke Kondo, ${ }^{2}$ and Yutai Katoh ${ }^{1}$ \\ 1. Materials Science and Technology Division, Oak Ridge National Laboratory, Oak Ridge, TN USA \\ 2. Institute of Advanced Energy, Kyoto University, Japan
}

Silicon carbide $(\mathrm{SiC})$ possesses excellent radiation tolerance, thus, $\mathrm{SiC}$ and its composites are promising materials for current and future nuclear systems [1]. However, the atomistic processes that underlie the irradiation response are not sufficiently understood, due largely to the limited spatial resolution of conventional analytical tools. Comparing aberration-corrected scanning transmission electron microscopy (AC-STEM) images with STEM image simulations of candidate defect structures is a method for analyzing individual defects in unprecedented detail. Conventional TEM methods can identify defects, such as dislocation loops and black dot damage (Figure 1; 10 dpa neutrons at $800^{\circ} \mathrm{C}$ ), but cannot provide insight regarding the exact atomistic details of the defects present.

Experiments were performed using an FEI Titan AC-STEM operated at $300 \mathrm{kV}$ and 30 mrad convergence angle, and image simulations were performed using the software QSTEM [2]. AC-STEM images of unirradiated, twinned $\beta$-SiC acquired in bright field $(\mathrm{BF})$, high-angle annular dark field (HAADF), and annular bright field (ABF) modes (Figure 2) are compared to simulations of a $60^{\circ}<111>$ boundary aligned along the [101] direction (Figure 2, insets). The close match between experiment and theory indicates AC-STEM coupled with image simulation is indeed a strong tool for identifying the atomistic character of defects. The matches are not perfect, however, and refinement is ongoing.

The nature of interstitial loops in irradiated $\mathrm{SiC}$ remains controversial: interstitial loops consisting of carbon atoms alone are hypothesized [3], whereas stoichiometric SiC loops are also hypothesized [4]. Candidate loop structures [5-6] of $\mathrm{C}$ and $\mathrm{SiC}<111>$ loops were simulated (Figure 3). Based upon the defocus simulations, identification of $\mathrm{SiC}$ vs. C-only loops should be possible using AC-STEM imaging.

Currently, we are producing ion-irradiated $\beta$-SiC specimens at doses and temperatures to maximize interstitial loop evolution. Using the guidance of these simulations, we anticipate direct observation of the nature of the loops and to identify the loops' stoichiometry. These results will be reported [7].

[1] Y. Katoh et al., Curr. Opin. Solid State Mater. Sci., 16[3] (2012) p. 143.

[2] http://elim.physik.uni-ulm.de/ ; C.T. Koch, Ph.D. thesis, Arizona State University (2002).

[3] Chao et al., Acta Mater. 62 (2014) p. 162.

[4] S. Kondo et al., in Mechanical Properties and Performance of Engineering Ceramics and Composite III, eds. E. Lara-Curzio et al. (Wiley, 2007) p. 91.

[5] T. Yano, J. Ceram. Soc. Japan, 111[3] (2003) p. 155.

[6] F. W. Clinard and L. W. Hobbs, in Phys. of Rad. Effects in Crystals, eds. Johnson \& Orlov, p. 387

[7] Research sponsored by Office of Fusion Energy Sciences (YK, CMP) and Nuclear Energy

University Partnerships (TK), US Department of Energy. Research supported as part of a user proposal through ORNL's Center for Nanophase Materials Sciences (CNMS), which is sponsored by the Scientific User Facilities Division, Office of Basic Energy Sciences, U.S. Department of Energy. 
Figure 1: 10 dpa irradiated $\beta$-SiC. (a) Dark field and (b) HR-TEM.
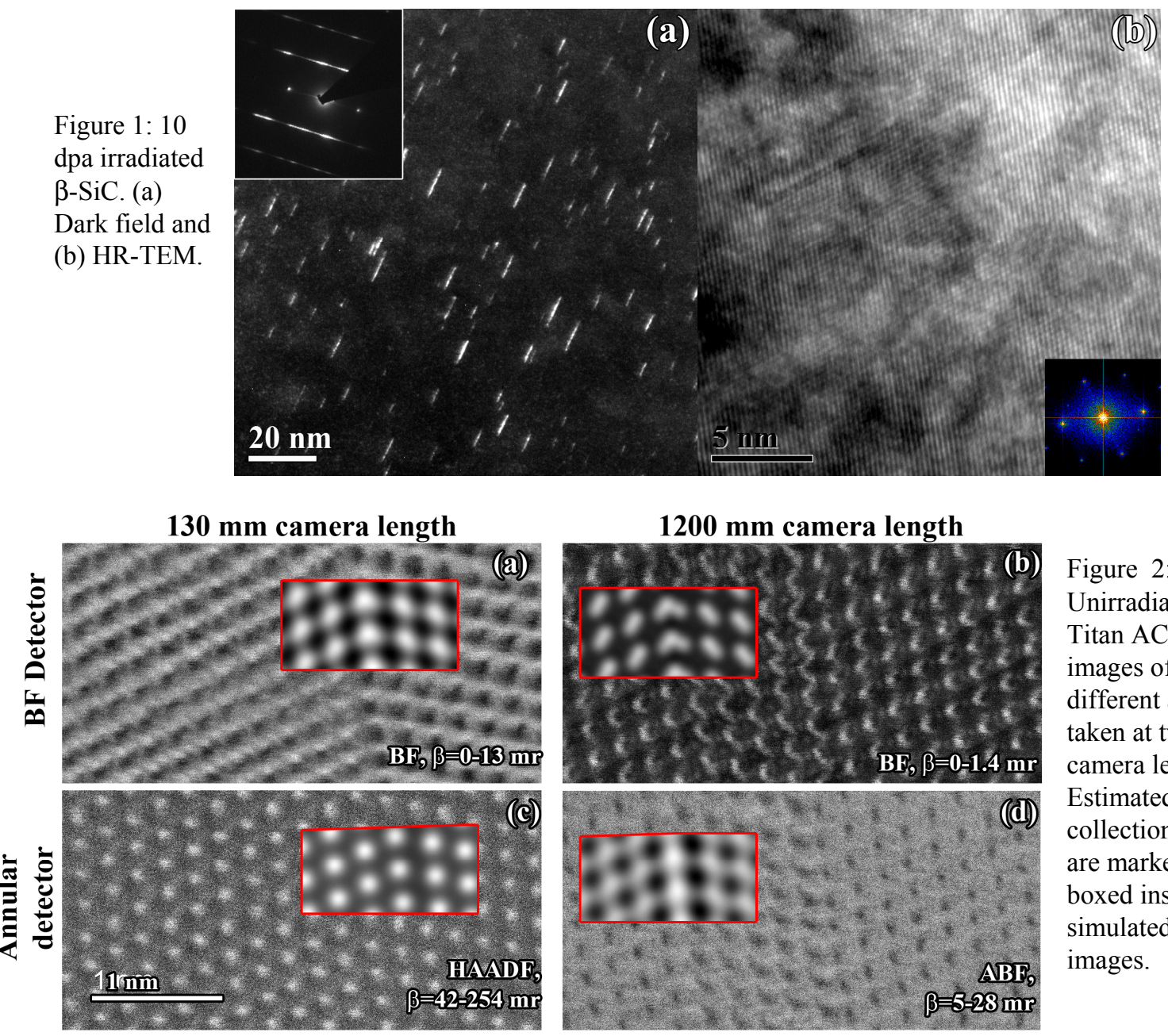

Figure 2:

Unirradiated $\beta$-SiC, Titan AC-STEM images of two different areas taken at two camera lengths.

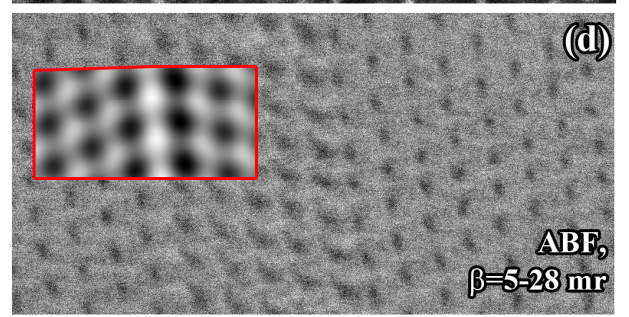
Estimated collection angles are marked. Redboxed insets are simulated STEM images.

Figure 3:

Left: simple model of a $5 \mathrm{~nm}$ (11-1) stoichiometric interstitial loop in a $10 \mathrm{~nm}$ slab of [101]-oriented SiC. Right: Montage of simulated images for stoichiometric or C-only loops under different imaging conditions.
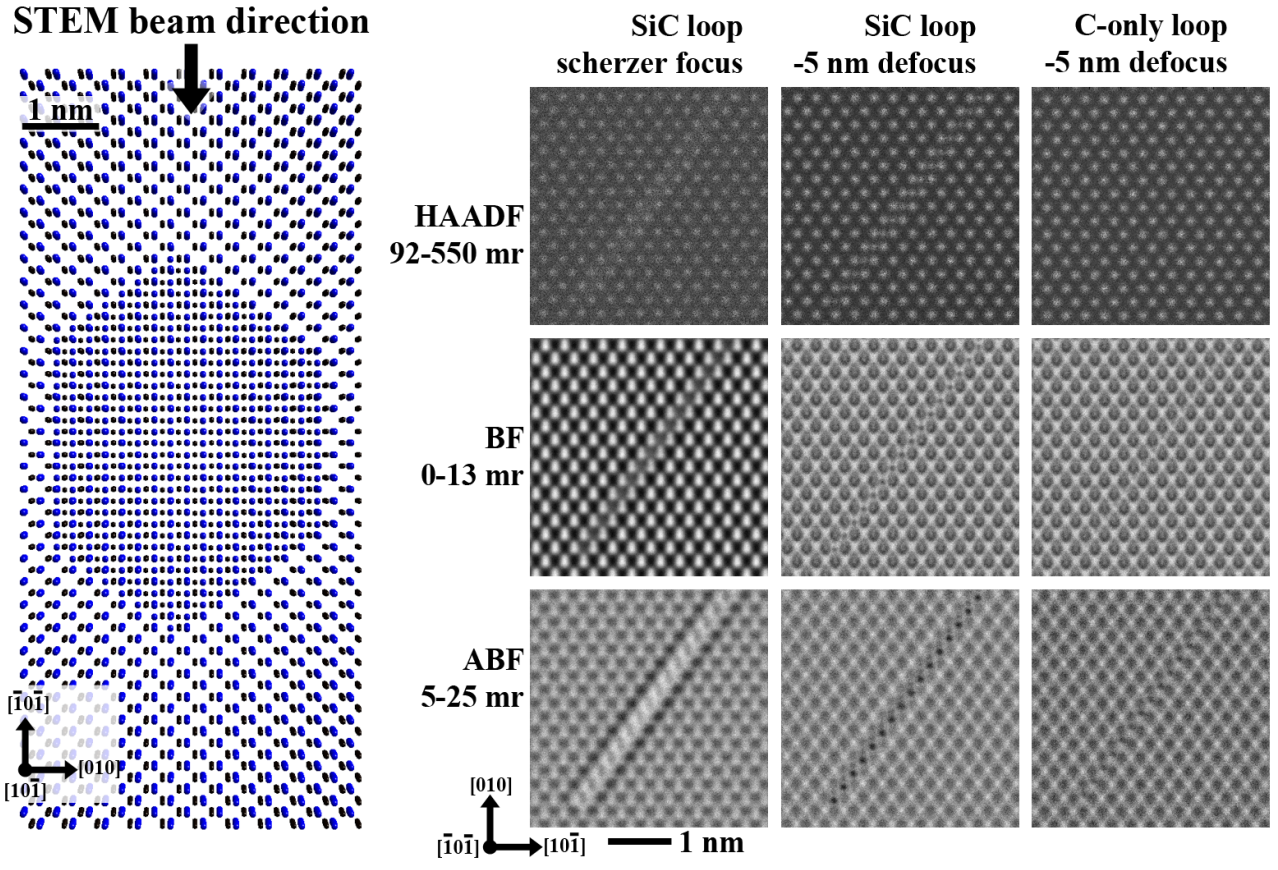